\title{
Nitroxoline as a promising alternative drug for the treatment of Lyme disease based on an in-vitro study
}

\author{
Hector S. Alvarez-Manzo ${ }^{1}$, Yumin Zhang ${ }^{1}$, Wanliang Shi ${ }^{1}$ and Ying Zhang ${ }^{2, *}$ \\ 1 Department of Molecular Microbiology and Immunology, Bloomberg School of Public Health, Johns Hopkins University, Baltimore, MD 21205, USA; \\ halvare3@jhu.edu (H.S.A.-M.); yzhan424@jhmi.edu (YM.Z.); wshi3@jhu.edu (W.S.); halvare3@jhu.edu (H.S.A.-M.); yzhan424@jhmi.edu (YM.Z.); \\ wshi3@jhu.edu (W.S.) \\ 2 State Key Laboratory for the Diagnosis and Treatment of Infectious Diseases, The First Affiliated Hospital, Zhejiang University School of Medicine, Hangzhou, \\ Zhejiang 310003, China \\ * Correspondence: yzhang207@zju.edu.cn
}

\begin{abstract}
Lyme disease (LD) is the most common vector-borne disease in USA and Europe and is caused by Borrelia burgdorferi. Despite proper treatment, approximately one fifth of patients will develop post-treatment LD syndrome (PTLDS), a condition which is poorly understood. One of the possible causes is thought to be due to persister forms of B. burgdorferi that are not effectively killed by the current Lyme antibiotics. In this study, we evaluated nitroxoline, an antibiotic used to treat urinary tract infections, for its activity against a stationary-phase culture enriched with persister forms of B. burgdorferi. Nitroxoline was found to be equivalent in activity against $B$. burgdorferi to cefuroxime (standard Lyme antibiotic) in different experiments. Moreover, we found that the three-drug combination cefuroxime + nitroxoline + clarithromycin eradicated $98.3 \%$ of stationary phase bacteria in the drug-exposure experiment and prevented the regrowth in the subculture study after drug exposure, as well as two-drug combinations cefuroxime + nitroxoline and clarithromycin + nitroxoline. These drug combinations should be further evaluated in a LD mouse model to assess if eradication of persister forms of B. burgdorferi in-vivo is possible and if so, whether nitroxoline could be repurposed as an alternative drug for the treatment of LD.
\end{abstract}

Keywords: Lyme disease, PTLDS, Borrelia burgdorferi, nitroxoline, drug repurposing

\section{Introduction}

Lyme disease (LD) is caused by the spirochete Borrelia burgdorferi sensu lato complex and the spectrum of clinical manifestations is wide, ranging from mild symptoms like fatigue, headache and fever, to severe manifestations such as arthritis, carditis, encephalopathy, encephalomyelitis, and neuropathy. The number of reported cases has increased significantly in the last two decades [1]. Nowadays, there are around 30,000 cases reported in USA every year [1] and in Europe, the estimated number of cases is around 65,000 [2] with the majority in Germany [3], Austria, Lithuania, Slovenia and Sweden [4]. Nevertheless, since LD is difficult to diagnose, it is commonly underreported [5] and total numbers could be at least 10 times higher [6,7]. For this reason, it is considered a major public health problem and the most important vector-borne disease in North America [4] and Europe [2]. Treatment for the early-stage disease is administered orally with doxycycline, amoxicillin or cefuroxime. In comparison, intravenous ceftriaxone is the preferred choice for late stage manifestations [8,9]. Unfortunately, antibiotic treatment with these drugs fails in 10-20\% of cases [10,11]. As a result, patients report fatigue, musculoskeletal pain [10] and some neurological symptoms like headaches and memory loss [12]. This condition is called "posttreatment Lyme disease syndrome" (PTLDS) and patients affected will see their quality of life diminished [12] with symptoms lasting for at least 6 months or longer after treatment [10,13]. PTLDS is nowadays a public health problem in USA and mathematical models calculate that the cumulative prevalence of PTLDS patients is actually around 2 million cases by year 2020 [14]. While the cause for this condition is unclear, one of the theories is that PTLDS could be due to persistent infection with persister forms of $B$. burgdorferi $[10,15]$. Several in-vivo animal studies have led to this theory by different techniques [16-18], and there is even a study that reported the presence of $B$. burgdorferi aggregates in the midgut of ticks after bloodfeeding with live-imaging, suggesting that infection with other forms than the spirochetal form in-vivo is possible [19].

In-vitro [20-23] and in-vivo [15] studies have shown that persister forms of B. burgdorferi (round-body and microcolony forms) are not easily cleared by the current Lyme antibiotics. Instead, we identified daptomycin as an antibiotic with anti-persister activity against $B$. burgdorferi and that the three-drug combination of cefuroxime + doxycycline + daptomycin is the most effective drug combination available for killing B. burgdorferi persisters both in-vitro [24,25] and in-vivo [15]. However, since daptomycin is an expensive parenteral drug, it is important to identify other drugs that are both more economically accessible and that can be orally administered.

In our recent study, we found that nitroxoline as a single drug, is equally effective against $B$. burgdorferi stationary-phase cultures enriched with persister forms, when compared with cefuroxime [26]. Nitroxoline (8-hydroxy-5-nitroquinoline) is an FDAapproved oral bacteriostatic antibiotic $[27,28]$ that is commercially available in Europe $[29,30]$ and has been used in Germany and other eastern European countries for the treatment of urinary tract infections [31]. However, in recent years, there is interest in the repurposing of this antibiotic as an anticancer drug [32-36] and also for the treatment of other infections [37-39]. Thus, our previous in-vitro results in addition to the studies that are looking into the possibility of repurposing this antibiotic, support the importance of performing further drug combination testing with nitroxoline in order to establish if this drug could be a useful candidate for the treatment of LD. To our knowledge, this is the first study that evaluates nitroxoline against a stationary-phase culture enriched with persister forms of $B$. burgdorferi. 


\section{Materials and Methods}

\section{Strain, media and culture}

Barbour-Stoenner-Kelly-H (BSK-H) medium (HiMedia Laboratories Pvt Ltd.) was filter-sterilized with $0.2 \mu \mathrm{m}$ filters and supplemented with $6 \%$ rabbit serum (Sigma-Aldrich, St. Louis, MO, USA) for the culturing of B. burgdorferi N40 strain. Cultures were incubated in $15 \mathrm{~mL}$ conical tubes in a microaerophilic incubator $\left(33^{\circ} \mathrm{C}, 5 \% \mathrm{CO} 2\right)$ for $7-10$ days in order to reach stationary phase, equivalent to 107 108 spirochetes/mL [20]. For the evaluation of drugs, a 96-well plate was used. The stationary-phase culture (100 $\mu \mathrm{L})$ was added to each well of the 96 well plate and drugs were added at the desired concentration. Afterwards, the plates were sealed and placed in a microaerophilic incubator for seven days at $33{ }^{\circ} \mathrm{C}$.

\section{Drugs}

Drugs used in this study were purchased from Sigma-Aldrich (St. Louis, MO, USA) and were dissolved in the appropriate solvents. The following drugs were utilized based on our previous studies [26]: Artemisinin (Arte), Cefuroxime (CefU), Clarithromycin (Clari), Clofazimine (CFZ), Daptomycin (Dapto), Doxycycline (Doxy), Erythromycin (Ery), Linezolid (LNZ), Nitazoxanide (NTZ), Nitroxoline (NTX), Rifabutin (Ribu). We included only drugs approved by the FDA and that can be administered orally (with the exception of Dapto which is a parenteral drug used in combination with CefU and Doxy as the three-drug control). The decision to utilize this drug is based on previous studies conducted by our research group that showed its high anti-borrelia persister activity [20,21,26]. Stock solutions were stored at $-20^{\circ} \mathrm{C}$.

\section{Microscopy}

B. burgdorferi samples were stained with the SYBR Green I/ Propidium Iodide (PI) assay and the bacterial viability was calculated with the green/red fluorescence ratio as previously described [40]. The SYBR Green I/ PI stock solution consisted of $10 \mu \mathrm{L}$ SYBR Green I (10,000x stock, Invitrogen) and $30 \mu \mathrm{L}$ PI $(20 \mu \mathrm{M}$, Sigma Aldrich) into $960 \mu \mathrm{L}$ sterile dH2O. For the work solution, the stock was diluted in a 1:4 ratio and for this study in particular, $20 \mu \mathrm{L}$ of the SYBR Green I/ PI work solution and $40 \mu \mathrm{L}$ of sample were added to each well to image under a BZ-X710 all-in-one fluorescence microscope (KEYENCE, Itasca, IL, USA). Three biological replicates were assessed for each drug combination and a representative picture for each sample was acquired. For the analysis of the samples, the ImageJ program was used in order to facilitate the counting of bacterial cells stained green or red and the bacterial viability of each sample was calculated by dividing the green fluorescence over the green and red fluorescence $(\mathrm{Green} /(\mathrm{Green}+\mathrm{Red}))$.

\section{Drug susceptibility testing}

Minimum inhibitory concentration (MIC) was determined with the microdilution method and bacterial growth inhibition was assessed after 7 days of drug addition by fluorescence microscope, after samples were stained with SYBR Green/PI. For the dose-response curves with CefU or NTX, a 7-day old B. burgdorferi stationary-phase culture was used and drugs were added at the desired concentration and incubated for 7 days. After this period of time, bacterial viability was assessed using the SYBR Green/PI assay. For both drugs, the Cmax concentration (Table 1) was used as the reference to choose the rest of the doses needed for the dose-response curve. Thus, in the case of CefU, the following concentrations were evaluated: untreated, 0.7, 1.75, 3.5, 7.0 (Cmax), 17.5, 35 and 70 $\mu \mathrm{g} / \mathrm{mL}$. For NTX, following concentrations were assessed: untreated, 0.56, 1.4, 2.8, 5.6 (Cmax), 14, 28 and $56 \mu \mathrm{g} / \mathrm{mL}$.

Drug-exposure experiments with drug combinations were assessed in the same way. We used a standard concentration of 5 $\mu \mathrm{g} / \mathrm{mL}$ as a close average Cmax concentration based on the literature, and selected drug combinations were further assessed at Cmax concentrations (Table 1). Moreover, the drug combination CefU + Doxy + Dapto was used as the positive drug control based on previous studies that showed that the combination with a cephalosporin + Doxy + Dapto is able to eradicate all borrelia bacteria including persister forms, it inhibits regrowth in the subculture study and achieves the best results in the drug-exposure experiments [23-26]. It is important to mention that Cmax concentrations were used to simulate the peak concentration of drugs in serum after oral ingestion.

Table 1. Cmax concentrations for drugs utilized in this study. In some cases, the concentration of drugs was converted from micromolar to $\mu \mathrm{g} / \mathrm{mL}$. In this study drugs were tested at their mean Cmax value.

\begin{tabular}{ccc}
\hline Drug & Cmax mean in $\boldsymbol{\mu g} / \mathbf{m L}$ (range) & Source \\
\hline Arte & 0.43 & {$[41]$} \\
CefU & 7.0 & {$[42]$} \\
Clari & 6.8 & {$[43]$} \\
CFZ & $1.1(0.7-1.4)$ & {$[44]$} \\
Dapto & 77.5 & {$[45]$} \\
Doxy & $2.6(1.5-3.6)$ & {$[46]$} \\
Ery & 3.0 & {$[47]$} \\
LNZ & $21.2(15.4-27.0)$ & {$[48]$} \\
NTZ & $10.6(8.6-12.6)$ & {$[49]$} \\
NTX & $5.6(2.45-8.75)$ & {$[50]$} \\
Ribu & $0.4(0.2-0.6)$ & {$[51]$} \\
\hline
\end{tabular}


Subculture study

Selected drug combinations were also evaluated in a subculture study to prove bacterial eradication. For this part, drug combinations were added at Cmax concentration to $1 \mathrm{~mL}$ of a 7-day old B. burgdorferi culture and placed in the incubator for 7 days. Then, samples were spun down twice to remove the antibiotic. After the second wash, cell pellet was resuspended with $500 \mu \mathrm{L}$ fresh BSK-H medium and $50 \mu \mathrm{L}$ were taken to inoculate $1 \mathrm{~mL}$ fresh BSK-H to proceed with the subculture as previously described [52]. After 3 weeks, samples were evaluated for bacterial regrowth with the SYBR Green/PI assay under the microscope. All combinations tested in the subculture study were assessed in triplicates.

\section{Statistical analysis}

The statistical analysis was carried out with the GraphPad Prism 9 software. In the case of the drug-exposure experiments, single drugs were compared with CefU as the positive drug control. For two-drug combinations, the positive drug control taken in this study was CefU + Doxy. As mentioned before, the positive three-drug control was CefU + Doxy + Dapto. The Kruskal-Wallis test was used to determine statistical differences and a p-value of less than 0.05 was considered significant.

\section{Results}

\section{MIC testing and dose-response curves}

The first aim of this study was to determine the MIC value for nitroxoline (NTX) and other control drugs (Table 2). The concentration that inhibited visible bacterial growth in the case of NTX was $1.25 \mu \mathrm{g} / \mathrm{mL}$. In the case of cefuroxime (CefU), the MIC value was 0.08 $\mu \mathrm{g} / \mathrm{mL}$. We also determined the IC50 for both drugs in a 7-day old stationary-phase culture of B. burgdorferi (Figure 1). The IC50 for CefU was of $7.9 \mu \mathrm{g} / \mathrm{mL}$ and in comparison, NTX IC50 value was $5.3 \mu \mathrm{g} / \mathrm{mL}$.

Table 2. MIC values of NTX and control drugs. The MIC for each drug was tested with the microdilution method.

\begin{tabular}{cccc}
\hline Drug & MIC $(\boldsymbol{\mu g} / \mathbf{m L})$ & Drug & MIC $(\boldsymbol{\mu g} / \mathbf{m L})$ \\
\hline CefU & 0.08 & CFZ & 5.0 \\
Doxy & 0.15 & Dapto & $>10.0$ \\
NTX & 1.25 & Ery & 0.02 \\
Arte & 5.0 & LNZ & 5.0 \\
Azi & 0.6 & NTZ & 10.0 \\
Clari & 0.02 & Ribu & 5.0 \\
\hline
\end{tabular}

CefU

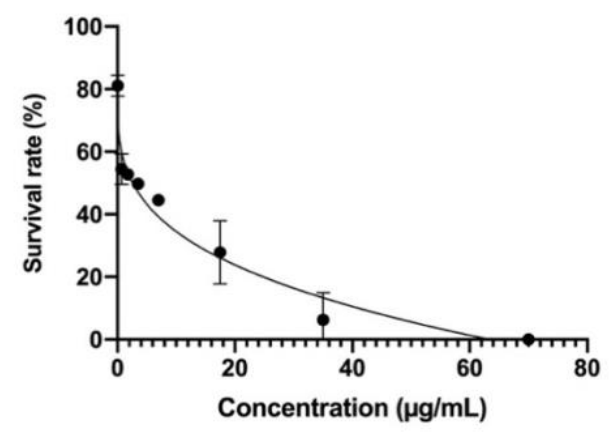

NTX

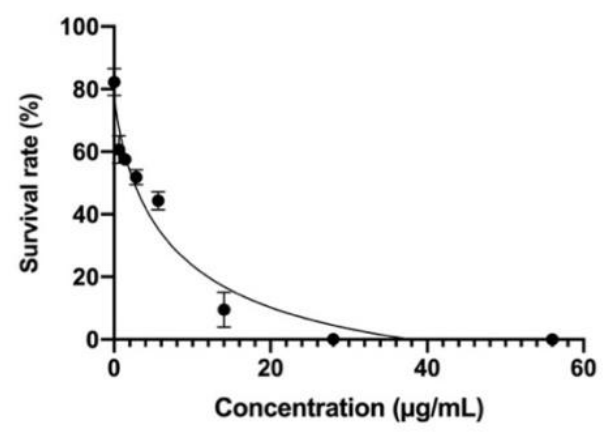

Figure 1. In-vitro drug-response curves for CefU and NTX in a 7-day old B. burgdorferi stationary-phase culture after a 7-day drug treatment. IC50 for CefU and NTX was $7.9 \mu \mathrm{g} / \mathrm{mL}$ and $5.3 \mu \mathrm{g} / \mathrm{mL}$ respectively. Concentrations used $(\mu \mathrm{g} / \mathrm{mL})$ : CefU: Untreated, 0.7, 1.75, 3.5, 7.0 (Cmax), 17.5, 35, 70; NTX: Untreated, 0.56, 1.4, 2.8, 5.6 (Cmax), 14, 28, 56. Abbreviations: cefuroxime (CefU), nitroxoline (NTX).

\section{Activity against stationary-phase culture at $5 \mu \mathrm{g} / \mathrm{mL}$ and Cmax concentration}

The next step was to perform a drug combination testing with nitroxoline and other drugs at a standard dose of $5 \mu \mathrm{g} / \mathrm{mL}$ for $7 \mathrm{days}$. Since the Cmax concentration for the different drugs is variable, we decided to take this concentration because it approaches the mean for the Cmax concentration of the drugs that we tested in this study. The stationary-phase B. burgdorferi had a survival rate of $36.3 \%$ upon 7-day treatment with Nitroxoline at $5 \mu \mathrm{g} / \mathrm{mL}$. In comparison, CefU had a slightly higher survival rate of $42.3 \%$. We then tested NTX in two- and three-drug combinations at $5 \mu \mathrm{g} / \mathrm{mL}$ in order to determine which combinations had a better activity against stationaryphase B. burgdorferi. We assessed a total of 19 two-drug combinations and 31 three-drug combinations at this standard concentration (Table 3). As mentioned before, the objective of this experiment was to select those concentrations that showed a promising result, in order to conduct a further experiment at Cmax concentration. Originally, we set up the threshold to determine a promising drug 
bioRxiv preprint doi: https://doi.org/10.1101/2021.02.04.429852; this version posted February 5,2021 . The copyright holder for this preprint (which was not certified by peer review) is the author/funder, who has granted bioRxiv a license to display the preprint in perpetuity. It is made available under aCC-BY-NC-ND 4.0 International license.

combination at $10 \%$ survival after treatment. Those three-drug combinations that produced bacterial viability higher than $10 \%$ would be discarded and not further evaluated. However, $87 \%$ of the combinations tested with NTX (27 out of 31) maintained bacterial viability at $10 \%$ or lower (Table 3 ).

Table 3. B. burgdorferi viability (in \%) in a 7-day old stationary-phase culture after a 7-day drug-exposure with single drugs, two-drug and three-drug combinations with CefU or NTX at $5 \mu \mathrm{g} / \mathbf{m L}$. Combinations in bold and underlined were further assessed at Cmax concentration (Table 4). A crossed line means values for these combinations were not evaluated. Abbreviations: untreated control (Cntrl), cefuroxime (CefU), doxycycline (Doxy), nitroxoline (NTX), artemisinin (Arte), azithromycin (Azi), clarithromycin (Clari), clofazimine (CFZ), erythromycin (Ery), linezolid (LNZ), nitazoxanide (NTZ), rifabutin (Ribu).

\begin{tabular}{|c|c|c|c|c|c|c|c|c|c|c|c|c|}
\hline & Ctrl & Doxy & CefU & NTX & Arte & Azi & Clari & CFZ & Ery & LNZ & NTZ & Ribu \\
\hline & 89.5 & 56.9 & 42.3 & 36.3 & 47.2 & 48.1 & 43.6 & 49.8 & 47.9 & 47.3 & 51.0 & 56.2 \\
\hline CefU & & 42.5 & - & 23.5 & 38.3 & 39.9 & 37.3 & 37.9 & 40.3 & 42.3 & 38.6 & 36.9 \\
\hline NTX & & 38.8 & 23.5 & $\longrightarrow$ & 11.9 & 19.2 & 13.3 & 13.6 & 18.1 & 7.3 & 30.2 & 19.9 \\
\hline Arte + NTX & & - & 4.0 & - & - & 11.9 & 6.4 & 5.7 & 4.1 & 8.9 & 7.7 & 5.8 \\
\hline Clari + NTX & & - & $\underline{5.5}$ & $\longrightarrow$ & & - & - & 4.9 & - & $\underline{7.6}$ & $\underline{9.0}$ & 4.3 \\
\hline CFZ + NTX & & - & $\overline{8.3}$ & $\longrightarrow$ & & 6.0 & & - & 2.5 & $\overline{7.5}$ & $\overline{11.4}$ & 5.0 \\
\hline LNZ + NTX & & - & 2.7 & - & & 11.7 & & & 7.2 & - & $\underline{6.1}$ & 12.9 \\
\hline NTZ + NTX & & - & $\overline{7.7}$ & - & & 8.7 & & & 10.0 & & - & 8.6 \\
\hline Ribu + NTX & & - & $\overline{4.7}$ & - & & 4.2 & & & 5.8 & & & - \\
\hline
\end{tabular}

Thus, we decided to modify the criteria to decide which combinations to test at Cmax concentration. Based on the literature, we chose to further evaluate drugs with a Cmax concentration higher than $5 \mu \mathrm{g} / \mathrm{mL}$. In the case of CefU, NTX, Clari, LNZ and NTZ, their Cmax concentrations are above the standard dose chosen $(5 \mu \mathrm{g} / \mathrm{mL})$. Thus, we hypothesized that drug combinations with a Cmax above 5 $\mu \mathrm{g} / \mathrm{mL}$ would result in a better result than at the standard dose. We did not further evaluate combinations with Arte, Azi, CFZ, Ery and Ribu, since Cmax concentrations for these drugs are below $5 \mu \mathrm{g} / \mathrm{mL}$. We decided this rationale would determine which combinations to test at Cmax concentration.

Table 4. Bacterial viability (in \%) in a B. burgdorferi 7-day old stationary-phase culture after drug-exposure experiment at Cmax concentration after a 7-day treatment. A crossed line means values for these combinations were not evaluated. Combinations in bold and underlined were further assessed in a subculture study (Figure 4, Table 6). CefU + Clari + NTX did not show statistical significance when compared to the three-drug control CefU + Doxy + Dapto and NTX and Clari did not show statistical significance when compared to CefU. In comparison, CefU + NTX, CefU + Clari and Clari + NTX showed statistical significance when compared to CefU + Doxy. Abbreviations: untreated control (Cntrl), cefuroxime (CefU), doxycycline (Doxy), nitroxoline (NTX), clarithromycin (Clari), daptomycin (Dapto), linezolid (LNZ), nitazoxanide (NTZ).

\begin{tabular}{cccccccc}
\hline & Cntrl & CefU & Doxy & NTX & Clari & LNZ & NTZ \\
\hline CefU & 95.6 & 38.0 & 64.2 & 37.6 & 40.2 & 48.7 & 52.2 \\
NTX & & - & 38.7 & $18.0^{*}$ & $25.1^{*}$ & 37.2 & 35.4 \\
CefU + Dapto & & 18.0 & 34.8 & - & $19.4^{*}$ & 30.0 & 33.9 \\
CefU + NTX & & - & 0.0 & - & - & - & $\underline{\mathbf{1 . 7}}$ \\
Clari + NTX & & - & - & - & $\underline{\mathbf{1 0 . 2}}$ & $\underline{\mathbf{9 . 8}}$ \\
LNZ + NTX & & - & - & - & - & $\underline{\mathbf{1 3 . 6}}$ & $\underline{\mathbf{1 4 . 3}}$ \\
\hline
\end{tabular}

* shows statistical significance.

We tested a total of 6 combinations at Cmax concentrations: CefU + Clari + NTX, CefU + LNZ + NTX, CefU + NTZ + NTX, Clari + LNZ + NTX, Clari + NTZ + NTX and LNZ + NTZ + NTX. Cmax concentrations for these drugs were taken from the literature and survival rates for these combinations at Cmax concentrations are depicted in Table 4. We compared the NTX three-drug combinations with the three-drug positive control CefU + Doxy + Dapto. The best combination was CefU + Clari + NTX, with a mean survival rate of $1.7 \%$ (Figure 2 and Figure 3). When performing the statistical analysis, NTX (survival rate 37.6\%) and Clari (survival rate 40.2\%) were not statistically significant when compared to CefU (survival rate 38.0\%). CefU + NTX (survival rate 18.0\%), CefU + Clari (survival rate 25.1\%) and Clari + NTX (survival rate 19.4\%) were statistically significant when compared to the two-drug control CefU + Doxy (survival rate 38.7\%). At last, CefU + Clari + NTX (survival rate 1.7\%) was not statistically significant when compared to threedrug control CefU + Doxy + Dapto (survival rate $0.0 \%$ ). 


\section{3-drug combo (Cmax)}

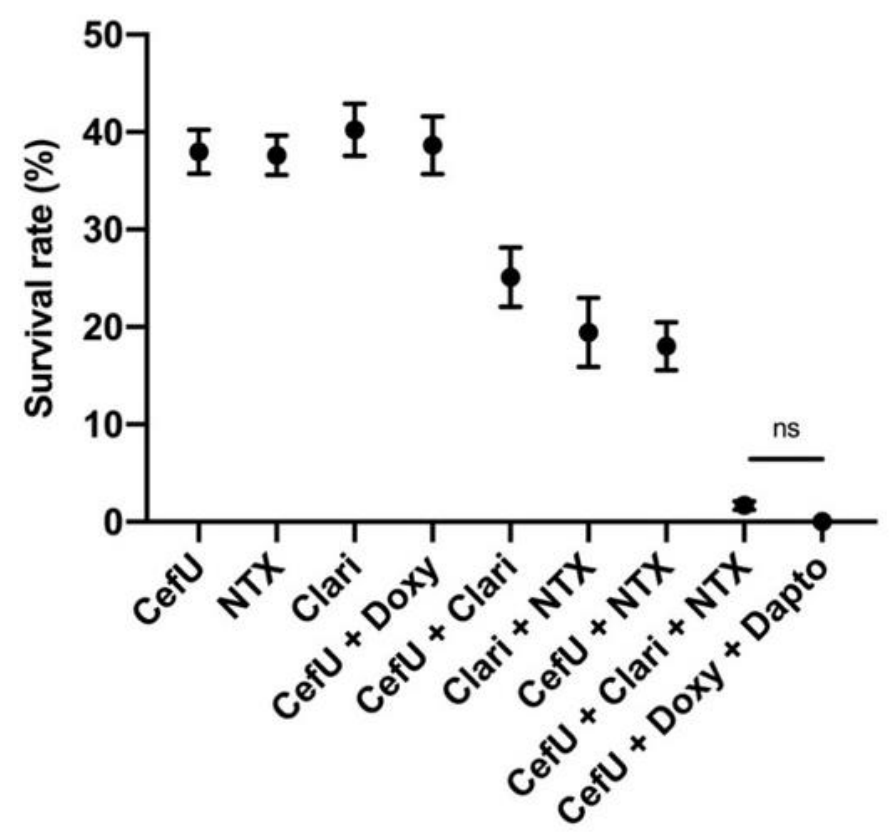

Figure 2. NTX-drug combinations of interest evaluated at Cmax concentration in a $B$. burgdorferi 7-day old stationary-phase culture after a 7-day treatment. CefU + Clari + NTX (survival rate 1.7\%) was not statistically significant when compared to the three-drug control CefU + Doxy + Dapto (survival rate 0.0\%). Abbreviations: cefuroxime (CefU), doxycycline (Doxy), nitroxoline (NTX), clarithromycin (Clari), daptomycin (Dapto). ${ }^{n s}$ no statistical significance.
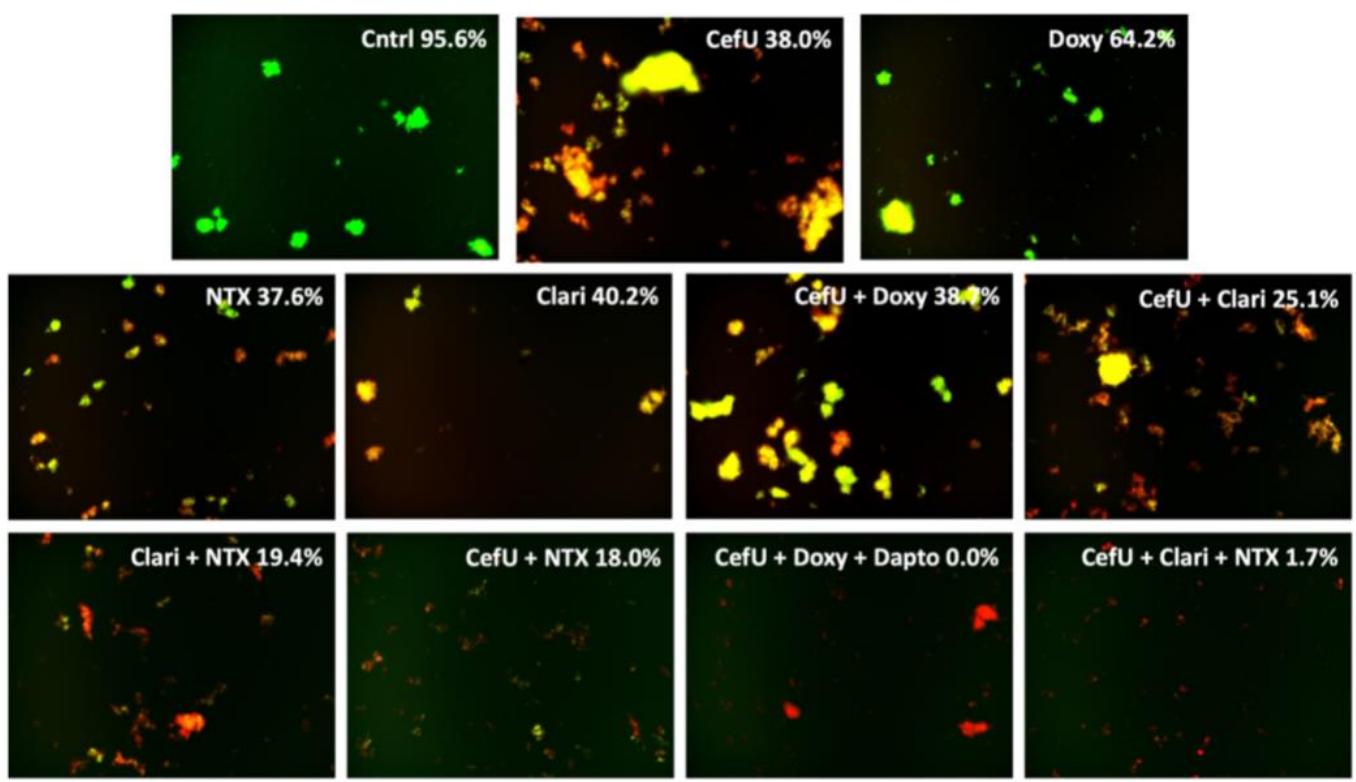

Figure 3. Microscope images for single drugs, two-drug and three-drug combinations of interest at Cmax concentration in a B. burgdorferi 7day old stationary-phase culture after a 7-day treatment. Abbreviations: untreated control (Cntrl), cefuroxime (CefU), doxycycline (Doxy), nitroxoline (NTX), clarithromycin (Clari), daptomycin (Dapto).

We then sought to compare the effect of drug combinations with NTX in comparison with drug combinations with CefU (standard Lyme antibiotic used for early disseminated and late disseminated disease) at Cmax concentrations. Thus, we chose those combinations tested at Cmax concentration that did not include CefU (Clari + LNZ + NTX, Clari + NTZ + NTX and LNZ + NTZ + NTX), replaced NTX with CefU and tested the following combinations: Clari + LNZ + CefU, Clari + NTZ + CefU and LNZ + NTZ + CefU. Survival rates for the comparison of these three-drug combinations are in Table 5. All six combinations, either with CefU or with NTX, showed to be better combinations when compared to the respective control (Table 5, left column). The best drug combinations were Clari $+\mathrm{NTZ}+$ CefU, Clari + LNZ + NTX and Clari + NTZ + NTX, since these kept bacterial viability at $12.6 \%, 13.6 \%$ and $14.3 \%$ respectively.

Table 5. Drug-exposure experiment with single drugs, two-drug and three-drug combinations directly comparing bacterial viability (in \%) of CefU and NTX in a 7-day old $B$. burgdorferi stationary-phase culture. A statistical analysis was performed comparing the performance of 
bioRxiv preprint doi: https://doi.org/10.1101/2021.02.04.429852; this version posted February 5, 2021. The copyright holder for this preprint (which was not certified by peer review) is the author/funder, who has granted bioRxiv a license to display the preprint in perpetuity. It is made available under aCC-BY-NC-ND 4.0 International license.

combinations with CefU and NTX to the control column. All combinations with CefU and NTX were statistically significant when compared to the respective control (left column). Abbreviations: untreated control (Cntrl), cefuroxime (CefU), nitroxoline (NTX), clarithromycin (Clari), linezolid (LNZ), nitazoxanide (NTZ).

\begin{tabular}{cccc}
\hline & Cntrl & CefU & NTX \\
\hline Clari & 95.6 & $38.0^{*}$ & $37.6^{*}$ \\
LNZ & 40.2 & $25.1^{*}$ & $19.4^{*}$ \\
NTZ & 58.7 & $37.2^{*}$ & $30.0^{*}$ \\
Clari + LNZ & 36.9 & $35.4^{*}$ & $33.9^{*}$ \\
Clari + NTZ & 42.0 & $12.6^{*}$ & $13.6^{*}$ \\
LNZ + NTZ & 46.8 & $38.1^{*}$ & $21.0^{*}$ \\
\hline * shows statistical significance. & & &
\end{tabular}

\section{Subculture study}

At last, we performed a subculture study to ensure bacterial killing by drug combinations (Figure 4, Table 6). We only tested CefU + Clari + NTX, since it was the only combination that achieved the best activity among other drug combinations tested with no statistical difference when compared to the three-drug positive control CefU + Doxy + Dapto. Both three-drug combinations achieved total eradication in the subculture study. In addition, the two drug combinations CefU + NTX and Clari + NTX also achieved eradication in this assay, despite not showing a very low survival ratio in the drug-exposure experiment (18.0\% and $19.4 \%$ respectively). However, single drugs Doxy, Cefu, Clari, NTX, and the two-drug controls CefU + Doxy and CefU + Clari showed regrowth after 3 weeks subculture study (Figure 4, Table 6).
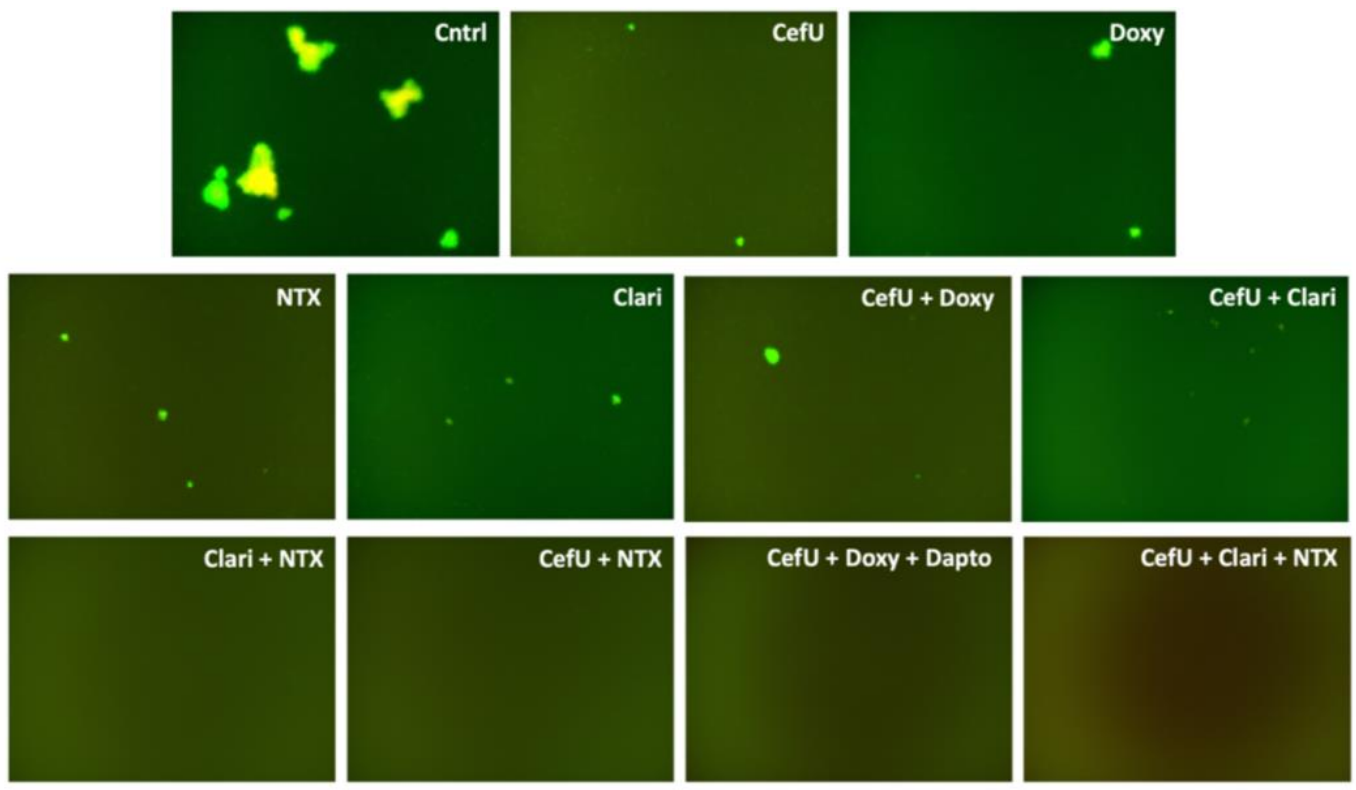

Figure 4. Microscope images for subcultures of single drugs, two-drug and three-drug combinations of interest. Abbreviations: untreated control (Cntrl), cefuroxime (CefU), doxycycline (Doxy), nitroxoline (NTX), clarithromycin (Clari), daptomycin (Dapto).

Table 6. Results of a 3-week subculture study for a 7-day old B. burgdorferi stationary-phase culture following a 7-day drug treatment at Cmax concentrations. Subcultures were assessed in triplicates. Each "+" (regrowth) or "_" (no regrowth) symbol represents the result for one replicate. CefU + NTX, Clari + NTX, CefU + Doxy + Dapto and CefU + Clari + NTX did not regrow after 3 weeks in the subculture experiment. Abbreviations: untreated control (Cntrl), cefuroxime (CefU), doxycycline (Doxy), nitroxoline (NTX), clarithromycin (Clari), daptomycin (Dapto), linezolid (LNZ), nitazoxanide (NTZ).

\begin{tabular}{cccc}
\hline Drug & Subculture results & Drug & Subculture results \\
\hline Cntrl & +++ & CefU + NTX & --- \\
CefU & +++ & Clari + NTX & --- \\
Doxy & +++ & CefU + Clari + NTX & --- \\
NTX & +++ & CefU + Doxy + Dapto & --- \\
Clari & +++ & & \\
CefU + Doxy & +++ & & \\
CefU + Clari & +++ & &
\end{tabular}




\section{Discussion}

We evaluated the performance of NTX compared to CefU at different concentrations against $B$. burgdorferi stationary-phase cultures. The goal of our study was to determine the in-vitro performance of NTX and compare it to the performance of the standard Lyme antibiotic CefU. The MIC test showed the MIC value for NTX was $1.25 \mu \mathrm{g} / \mathrm{mL}$, consistent with our previous finding [26], while CefU and Doxy MIC-values were $0.08 \mu \mathrm{g} / \mathrm{mL}$ for both, also consistent with previous results [53]. Moreover, the NTX MIC value is below its Cmax concentration $(5.6 \mu \mathrm{g} / \mathrm{mL})$, indicating it could reach growth inhibitory concentration when used in-vivo.

We were also interested in determining the effect of NTX in B. burgdorferi stationary-phase cultures enriched with persisters. In a previous study conducted by our research group [26], we established that NTX at Cmax achieved a similar eradication rate of a $B$. burgdorferi stationary-phase culture when compared to CefU also at Cmax. In the present study, CefU (survival rate 38.0\%) and NTX (survival rate 37.6) single-drug results in the drug-exposure experiment were consistent with our previous study [26]. Two-drug combinations CefU + NTX (survival rate 18.0\%), CefU + Clari (survival rate 25.1\%) and Clari + NTX (survival rate 19.4\%) were the best two-drug combinations and proved to be statistically significant when compared to the two-drug control CefU + Doxy (survival rate 38.7\%). In the case of the three-drug combinations assessed at Cmax (Table 4), CefU + Clari + NTX achieved a survival rate of $1.7 \%$ and showed no statistical significance compared with the three-drug control CefU + Doxy + Dapto (survival rate $0.0 \%$ ). This is particularly interesting since this is the first oral combination that we have identified to be equivalent towards the three-drug combination with CefU + Doxy + Dapto. Besides, it also makes sense that this combination in particular achieved the best result, since two-drug combinations that contain this very same drugs (CefU + NTX, CefU + Clari and Clari + NTX), achieved in this study the best two-drug results as previously mentioned. Thus, the combination of these three-drugs proved to be the strongest combination tested in this study and proved to be equivalent to the positive control. Moreover, CefU + Clari + NTX was also the only combination that reduced the survival rate when adjusting the concentration to Cmax. All other three-drug combination survival rates increased when compared with their $5 \mu \mathrm{g} / \mathrm{mL}$ result (Table 3 ).

Finally, we replaced CefU with NTX in some two-drug and three-drug combinations to compare the effect of each drug and determine which one could have a higher impact on persister forms of B. burgdorferi (Table 5). We found that combinations with either CefU or NTX were equally effective when compared to their control. Thus, although not better, it appears at least in-vitro that NTX has similar activity as the standard Lyme antibiotic CefU when used alone and in drug-combinations against stationary-phase cultures enriched with persister forms of $B$. burgdorferi.

In the subculture study (Table 6), the positive control CefU + Doxy + Dapto and the combination of interest CefU + Clari + NTX were shown to completely eradicate $B$. burgdorferi stationary-phase culture as demonstrated by lack of regrowth after 3 weeks of subculturing. Interestingly, not only these combinations achieved eradication, but CefU + NTX and Clari + NTX also did. This finding in particular suggests that it is not necessary for a drug-combination in vitro to achieve complete killing in the drug-exposure experiment, to be able to achieve eradication in the subculture study.

NTX has been described as a metal-chelator drug. Specifically, an in-vitro study showed that NTX dispersed Pseudomonas aeruginosa biofilm by chelating $\mathrm{Fe}^{2+}$ and $\mathrm{Zn}^{2+}$ ions [37]. Another study found that NTX had a high antimicrobial activity against Escherichia coli, Providencia rettgeri and Klebsiella pneumoniae [38]. Finally, biofilms formed by methicillin-resistant Staphylococcus aureus and Acinetobacter baumannii are also eradicated by NTX in-vitro [39]. In the stationary-phase cultures, as seen in Figure 3 (Cntrl survival rate $95.6 \%), B$. burgdorferi tends to form microcolonies, which is consistent with a previous study [24]. These B. burgdorferi microcolonies could be analogous to small biofilms, as $B$. burgdorferi is able to generate biofilms in-vitro [54]. Thus, since NTX does have a high activity against biofilms as mentioned previously, the activity of this drug against $B$. burgdorferi stationary-phase cultures is not surprising. However, it still remains to be determined as to how this drug might be eradicating B. burgdorferi. B. burgdorferi does not utilize iron for its metabolism. Instead, different studies have demonstrated that manganese and copper might have an important role in the metabolism of B. burgdorferi, since these are the cofactors necessary for molecules related to virulence [55], mammalian adaptation [56], survival [57,58], and persistence within the tick [59]. As mentioned before, NTX has shown to chelate $\mathrm{Zn}^{2+}$ from $P$. aeruginosa biofilm, achieving biofilm disruption in-vitro [37]. Thus, it is possible that NTX might be chelating zinc or other ions like manganese or copper and as a result, causing $B$. burgdorferi to die. This hypothesis still has to be proven with the addition and removal of zinc, manganese, copper and other metals from the BSK-H medium and the treatment with NTX in future studies.

NTX is an FDA-approved drug not commercially available in USA but it is clinically used in some European countries [2931]. Cephalosporin and tetracycline allergy prevalence among the general population ranges from $0.8-1.7 \%$ and $0.5-1.6 \%$ respectively and it is more frequent among women and the elderly population [60,61]. If further investigated, NTX could be repurposed for the treatment of acute stages of LD for those patients that do not tolerate treatment with the common Lyme antibiotics. In addition, our results regarding the activity of NTX as single drug and in combination (CefU + Clari + NTX), could potentially be useful in treating PTLDS due to persister forms of B. burgdorferi. We realize that the main limitation of this study is that we only carried out in-vitro experiments. However, as far as we are concerned, this is the first study to evaluate NTX in detail against $B$. burgdorferi, and our findings are completely new, promising and warrant future studies with NTX in cell lines and in animal models of $B$. burgdorferi infection. Moreover, we only tested NTX in the context of B. burgdorferi sensu stricto N40 strain. It will be relevant to evaluate if NTX has activity against Borrelia afzelii and Borrelia garinii, since these are the most prevalent genospecies that cause LD in Europe [6264], where NTX is commercially available. 

available under aCC-BY-NC-ND 4.0 International license.

\section{Conclusions}

We showed that NTX is as active as CefU in the MIC experiment, as well as against non-growing persister forms of B. burgdorferi. We found that the three-drug combination CefU + Clari + NTX was equivalent to the previous three-drug control with CefU + Doxy + Dapto. Since the combination with NTX found in this study involves only oral drugs (unlike the combination with the intravenous drug Dapto), we propose that this new combination could be considered as the new positive control for further in-vitro studies that evaluate drugs in the context of B. burgdorferi stationary-phase cultures. Moreover, CefU + Clari + NTX, as well as two-drug combinations CefU + NTX and Clari + NTX should be investigated in the future in mouse models to establish if these combinations are able to eradicate persister forms of B. burgdorferi in-vivo. Our results suggest that NTX, if further investigated and confirmed, could be repurposed for the treatment of active stages of LD. Moreover, it could also be useful for patients with PTLDS if the origin of PTLDS is caused by the presence of persister forms of B. burgdorferi. However, studies in cell lines and in-vivo experiments have to be carried out before repurposing this drug as an alternative. Finally, the elucidation of the mechanism by which NTX eradicates B. burgdorferi is key to better understanding the survival strategies of B. burgdorferi persisters and also for identification of new antimicrobial targets.

Author Contributions: Conceptualization, H.S.A.-M. and Y.Z. (Ying Zhang); methodology, H.S.A.-M. and Y.Z. (Yumin Zhang); formal analysis, H.S.A.-M.; data curation, H.S.A.-M.; writing—original draft preparation, H.S.A.-M.; writing—review and editing, Y.Z. (Ying Zhang); supervision, W.S.; funding acquisition, Y.Z. (Ying Zhang). All authors have read and agreed to the published version of the manuscript.

Funding: This research received funding from the Global Lyme Alliance, the Steven and Alexandra Cohen Foundation, the LivLyme Foundation, NatCapLyme, and the Einstein-Sim Family Charitable Fund.

Acknowledgments: We acknowledge the support given by the Global Lyme Alliance, the Steven and Alexandra Cohen Foundation, the LivLyme Foundation, NatCapLyme, and the Einstein-Sim Family Charitable Fund.

Conflicts of Interest: The authors declare no conflict of interest.

\section{References}

1. Centers for Disease Control and Prevention (CDC), Lyme Disease Home. Available online: https://www.cdc.gov/lyme/index.html (accessed on 9 December 2020).

2. Semenza, J.C.; Suk, J.E. Vector-Borne Diseases and Climate Change: a European Perspective. FEMS Microbiol. Lett. 2018, 365 , fnx244. https://doi.org/10.1093/femsle/fnx244

3. Enkelmann, J.; Böhmer, M.; Fingerle, V.; Siffczyk, C.; Werber, D.; Littmann, M.; Merbecks, S.-S.; Helmeke, C.; Schroeder, S.; Hell, S.; Schlotthauer, U.; Burckhardt, F.; Stark, K.; Schielke, A.; Wilking, H. Incidence of Notified Lyme Borreliosis in Germany, 2013-2017. Sci. Rep. 2018, 8, 14976. https://doi.org/10.1038/s41598-018-33136-0

4. Borchers, A.T.; Keen, C.L.; Huntley, A.C.; Gershwin, M.E. Lyme Disease: A Rigorous Review of Diagnostic Criteria and Treatment. J. Autoimmun. 2015, 57, 82-115. https://doi.org/10.1016/j.jaut.2014.09.004

5. Centers for Disease Control and Prevention (CDC), Summary of Notifiable Diseases — United States, 2010. Available online: https://www.cdc.gov/mmwr/preview/mmwrhtml/mm5953a1.htm (accessed on 8 December 2020).

6. Kuehn, B.M. CDC Estimates 300000 US Cases of Lyme Disease Annually. JAMA 2013, 310, 1110. https://doi.org/10.1001/jama.2013.278331

7. Nelson, C.A.; Saha, S.; Kugeler, K.J.; Delorey, M.J.; Shankar, M.B.; Hinckley, A.F.; Mead, P.S. Incidence of Clinician-Diagnosed Lyme Disease, United States, 2005-2010. Emerg. Infect. Dis. 2015, 21, 1625-31. https://doi.org/10.3201/eid2109.150417

8. Halperin, J.J.; Shapiro, E.D.; Logigian, E.; Belman, A.L.; Dotevall, L.; Wormser, G.P.; Krupp, L.; Gronseth, G.; Bever, C.T. Practice Parameter: Treatment of Nervous System Lyme Disease (an Evidence-Based Review): Report of the Quality Standards Subcommittee of the American Academy of Neurology. Neurology 2007, 69, 91-102. https://doi.org/10.1212/01.wnl.0000265517.66976.28

9. Wormser, G.P.; Dattwyler, R.J.; Shapiro, E.D.; Halperin, J.J.; Steere, A.C., Klempner, M.S.; Krause, P.J.; Bakken, J.S.; Strle, F.; Stanek, G.; Bockenstedt, L.; Fish, D.; Dumler, J.S.; Nadelman, R.B. The Clinical Assessment, Treatment, and Prevention of Lyme Disease, Human Granulocytic Anaplasmosis, and Babesiosis: Clinical Practice Guidelines by the Infectious Diseases Society of America. Clin. Infect. Dis. 2006, 43, 1089-134. https://doi.org/10.1086/508667

10. Aucott, J.N. Posttreatment Lyme Disease Syndrome. Infect. Dis. Clin. N. Am. 2015, 29, 309-23. https://doi.org/10.1016/j.idc.2015.02.012

11. Marques, A. Chronic Lyme Disease: A Review. Infect. Dis. Clin. N. Am. 2008, 22, 341-60. https://doi.org/10.1016/j.idc.2007.12.011

12. Eikeland, R.; Mygland, Å.; Herlofson, K.; Ljøstad, U. European Neuroborreliosis: Quality of Life 30 Months after Treatment. Acta Neurol. Scand. 2011, 124, 349-54. https://doi.org/10.1111/j.1600-0404.2010.01482.x

13. Center for Disease Control and Prevention (CDC), Post-Treatment Lyme Disease Syndrome. Available online: https://www.cdc.gov/lyme/postlds/index.html (accessed on 13 December 2020).

14. DeLong, A.; Hsu, M.; Kotsoris, H. Estimation of Cumulative Number of Post-Treatment Lyme Disease Cases in the US, 2016 and 2020. BMC Public Health 2019, 19, 352. https://doi.org/10.1186/s12889-019-6681-9

15. Feng, J.; Li, T.; Yee, R.; Yuan, Y.; Bai, C.; Cai, M.; Shi, W.; Embers, M.; Brayton, C.; Saeki, H.; Gabrielson, K.; Zhang, Y. Stationary Phase Persister/Biofilm Microcolony of Borrelia burgdorferi Causes More Severe Disease in a Mouse Model of Lyme Arthritis: Implications for Understanding Persistence, Post-Treatment Lyme Disease Syndrome (PTLDS), and Treatment Failure. Discov. Med. 2019, 27, 125-38.

16. Embers, M.E.; Barthold, S.W.; Borda, J.T.; Bowers, L.; Doyle, L.; Hodzic, E.; Jacobs, M.B.; Hasenkampf, N.R.; Martin, D.S.; Narasimhan, S.; Phillipi-Falkenstein, K.M.; Purcell, J.E.; Ratterree, M.S.; Phillip, M.T. Persistence of Borrelia burgdorferi in Rhesus Macaques Following Antibiotic Treatment of Disseminated Infection. PLOS ONE 2012, 7, e29914. https://doi.org/10.1371/journal.pone.0029914

17. Hodzic, E.; Imai, D.; Feng, S.; Barthold, S.W. Resurgence of Persisting Non-Cultivable Borrelia burgdorferi Following Antibiotic Treatment in Mice. PLOS ONE 2014, 9, e86907. https://doi.org/10.1371/journal.pone.0086907

18. Straubinger, R.K.; Summers, B.A.; Chang, Y.F.; Appel, M.J. Persistence of Borrelia burgdorferi in Experimentally Infected Dogs after Antibiotic Treatment. J. Clin. Microbiol. 1997, 35, 111-6. https://doi.org/10.1128/JCM.35.1.111-116.1997 
bioRxiv preprint doi: https://doi.org/10.1101/2021.02.04.429852; this version posted February 5, 2021. The copyright holder for this preprint (which was not certified by peer review) is the author/funder, who has granted bioRxiv a license to display the preprint in perpetuity. It is made available under aCC-BY-NC-ND 4.0 International license.

19. Dunham-Ems, S.M.; Caimano, M.J.; Pal, U.; Wolgemuth, C.W.; Eggers, C.H.; Balic, A.; Radolf, J.D. Live Imaging Reveals a Biphasic Mode of Dissemination of Borrelia burgdorferi within Ticks. J. Clin. Invest. 2009, 119, 3652-65. https://doi.org/10.1172/JCI39401

20. Feng, J.; Wang, T.; Shi, W.; Zhang, S.; Sullivan, D.; Auwaerter, P.G.; Zhang, Y. Identification of Novel Activity Against Borrelia burgdorferi Persisters using an FDA Approved Drug Library. Emerg. Microbes Infect. 2014, 3, 1-8. https://doi.org/10.1038/emi.2014.53

21. Feng, J.; Weitner, M.; Shi, W.; Zhang, S.; Sullivan, D.; Zhang, Y. Identification of Additional Anti-Persister Activity against Borrelia burgdorferi from an FDA Drug Library. Antibiotics 2015, 4, 397-410. https://doi.org/10.3390/antibiotics4030397

22. Feng, J.; Shi, W.; Zhang, S.; Zhang, Y. Identification of New Compounds with High Activity against Stationary Phase Borrelia burgdorferi from the NCI Compound Collection. Emerg. Microbes Infect. 2015, 4, e31. https://doi.org/10.1038/emi.2015.31

23. Feng, J.; Shi, W.; Zhang, S.; Sullivan, D.; Auwaerter, P.G.; Zhang, Y. A Drug Combination Screen Identifies Drugs Active against AmoxicillinInduced Round Bodies of In Vitro Borrelia burgdorferi Persisters from an FDA Drug Library. Front. Microbiol. 2016, 7 , 743. https://doi.org/10.3389/fmicb.2016.00743

24. Feng, J.; Auwaerter, P.G.; Zhang, Y. Drug Combinations against Borrelia burgdorferi Persisters In Vitro: Eradication Achieved by Using Daptomycin, Cefoperazone and Doxycycline. PLoS ONE 2015, 10, e0117207. https://doi.org/10.1371/journal.pone.0117207

25. Feng, J.; Zhang, S.; Shi, W.; Zhang, Y. Ceftriaxone Pulse Dosing Fails to Eradicate Biofilm-Like Microcolony B. Burgdorferi Persisters which are Sterilized by Daptomycin/ Doxycycline/Cefuroxime without Pulse Dosing. Front Microbiol 2016, $7,1744$. https://doi.org/10.3389/fmicb.2016.01744

26. Alvarez-Manzo, H.S.; Zhang, Y.; Shi, W.; Zhang, Y. Evaluation of Disulfiram Drug Combinations and Identification of Other More Effective Combinations against Stationary Phase Borrelia Burgdorferi. Antibiotics 2020, 9, 542. https://doi.org/10.3390/antibiotics9090542

27. Pelletier, C.; Prognon, P.; Bourlioux, P. Roles of Divalent Cations and pH in Mechanism of Action of Nitroxoline against Escherichia coli Strains. Antimicrob. Agents Chemother. 1995, 39, 707-13. https://doi.org/10.1128/AAC.39.3.707

28. Sakka, N.E.; Gould, I.M. Role of Old Antimicrobial Agents in the Management of Urinary Tract Infection. Expert Rev. Clin. Pharmacol. 2016, 9, 1047-56. https://doi.org/10.1080/17512433.2016.1189325

29. Kresken, M.; Körber-Irrgang, B. In Vitro Activity of Nitroxoline against Escherichia coli Urine Isolates from Outpatient Departments in Germany. Antimicrob. Agents Chemother. 2014, 58, 7019-20. https://doi.org/10.1128/AAC.03946-14

30. Wijma, R.A.; Huttner, A.; Koch, B.C.P.; Mouton, J.W.; Muller, A.E. Review of the Pharmacokinetic Properties of Nitrofurantoin and Nitroxoline. J. Antimicrob. Chemother. 2018, 73, 2916-26. https://doi.org/10.1093/jac/dky255

31. Hof, H.; Bertsch, D.; Passek, D.; Schwarz, R. Nitroxolin - eine Option zur antibiotischen Therapie von Harnwegsinfektionen. Urologe 2017, 56, 167-71. https://doi.org/10.1007/s00120-016-0218-y

32. Jiang, H.; Xing, J.; Wang, C.; Zhang, H.; Yue, L.; Wan, X.; Chen, W.; Ding, H.; Xie, Y.; Tao, H.; Chen, Z.; Jiang, H.; Chen, K.; Chen, S.; Zheng, M.; Zhang, Y.; Luo, C. Discovery of Novel BET Inhibitors by Drug Repurposing of Nitroxoline and its Analogues. Org. Biomol. Chem. 2017, 15, 9352-61. https://doi.org/10.1039/C7OB02369C

33. Mitrović, A.; Kos, J. Nitroxoline: Repurposing its Antimicrobial to Antitumor Application. Acta Biochim. Pol. 2019, 66, 521-31. https://doi.org/10.18388/abp.2019_2904

34. Xu, N.; Huang, L.; Li, X.; Watanabe, M.; Li, C.; Xu, A.; Liu, C.; Li, Q.; Araki, M.; Wada, K.; Nasu, Y.; Huang, P. The Novel Combination of Nitroxoline and PD-1 Blockade, Exerts a Potent Antitumor Effect in a Mouse Model of Prostate Cancer. Int. J. Biol. Sci. 2019, 15, 919-928. https://doi.org/10.7150/ijbs.32259

35. Zhang, Q.; Wang, S.; Yang, D.; Pan, K.; Li, L.; Yuan, S. Preclinical Pharmacodynamic Evaluation of Antibiotic Nitroxoline for Anticancer Drug Repurposing. Oncol Lett 2016, 11, 3265-72. https://doi.org/10.3892/ol.2016.4380

36. Veschi, S.; De Lellis, L.; Florio, R.; Lanuti, P.; Massucci, A.; Tinari, N.; De Tursi, M.; Di Sebastiano, P.; Marchisio, M.; Natoli, C.; Cama, A. Effects of Repurposed Drug Candidates Nitroxoline and Nelfinavir as Single Agents or in Combination with Erlotinib in Pancreatic Cancer Cells. J. Exp. Clin. Cancer Res. 2018, 37, 236. https://doi.org/10.1186/s13046-018-0904-2

37. Sobke, A.; Klinger, M.; Hermann, B.; Sachse, S.; Nietzsche, S.; Makarewicz, O.; Keller, P.M.; Pfister, W.; Straube, E. The Urinary Antibiotic 5Nitro-8-Hydroxyquinoline (Nitroxoline) Reduces the Formation and Induces the Dispersal of Pseudomonas aeruginosa Biofilms by Chelation of Iron and Zinc. Antimicrob. Agents Chemother. 2012, 56, 6021-25 https://doi.org/10.1128/AAC.01484-12

38. Cherdtrakulkiat, R.; Lawung, R.; Nabu, S.; Tantimavanich, S.; Sinthupoom, N.; Prachayasittikul, S.; Prachayasittikul, V. Nitroxoline: A Potent Antimicrobial Agent against Multidrug Resistant Enterobacteriaceae. EXCLI J. 2019, 18, 445-53. https://doi.org/10.17179/excli2019-1378

39. Abouelhassan, Y.; Yang, Q.; Yousaf, H.; Nguyen, M.T.; Rolfe, M.; Schultz, G.S.; Huigens 3rd, R.W. Nitroxoline: A Broad-Spectrum BiofilmEradicating Agent against Pathogenic Bacteria. Int. J. Antimicrob. Agents 2017, 49, 247-51. https://doi.org/10.1016/j.ijantimicag.2016.10.017

40. Feng, J.; Wang, T.; Zhang, S.; Shi, W.; Zhang, Y. An Optimized SYBR Green I/PI Assay for Rapid Viability Assessment and Antibiotic Susceptibility Testing for Borrelia Burgdorferi. PLoS ONE 2014, 9, e111809. https://doi.org/10.1371/journal.pone.0111809

41. Artemisinin Available online: http://www.antimicrobe.org/drugpopup/artemisinin.htm (accessed on 11 November 2020).

42. Cefuroxime Available online: http://www.antimicrobe.org/drugpopup/Cefuroxime.htm (accessed on 11 November 2020).

43. Clarithromycin Available online: http://www.antimicrobe.org/drugpopup/clarithromycin.htm (accessed on 11 November 2020).

44. Yawalkar, S.J.; Vischer, W. Lamprene (Clofazimine) in Leprosy. Lepr. Rev. 1979, 50, 135-44. https://doi.org/10.5935/0305-7518.19790020

45. Daptomycin. Available online: http://antimicrobe.org/drugpopup/Daptomycin.pdf (accessed on 11 November 2020).

46. Doxycycline. Available online: http://www.antimicrobe.org/drugpopup/Doxycycline.htm (accessed on 11 November 2020).

47. Erythromycin. Available online: http://www.antimicrobe.org/drugpopup/Erythromycin.pdf (accessed on 11 November 2020).

48. Linezolid and Other Oxazolidinones. Available online: http://www.antimicrobe.org/d13.asp (accessed on 11 November 2020).

49. Food and Drug Administration (FDA) Prescribing Information for Alinia®. Available online: https://www.accessdata.fda.gov/drugsatfda_docs/label/2005/021818lbl.pdf (accessed on 11 November 2020).

50. Bergogne-Berenzin, E.; Berthelot, G.; Muller-Serieys, C. Present Status of Nitroxoline. Pathol. Biol. 1987, 35, 873-8.

51. Rifabutin for Mycobacterial Infections. Available online: http://www.antimicrobe.org/drugpopup/Rifabutin-myco.htm (accessed on 11 November 2020).

52. Feng, J.; Weitner, M.; Shi, W.; Zhang, S.; Zhang, Y. Eradication of Biofilm-Like Microcolony Structures of Borrelia Burgdorferi by Daunomycin and Daptomycin but Not Mitomycin $\mathrm{C}$ in Combination with Doxycycline and Cefuroxime. Front. Microbiol. 2016,7 , 62. https://doi.org/10.3389/fmicb.2016.00062

53. Hunfeld, K.-P.; Brade, V. Antimicrobial Susceptibility of Borrelia burgdorferi Sensu Lato: What We Know, What We Don't Know, and What We Need to Know. Wien. Klin. Wochenschr. 2006, 118, 659-68. https://doi.org/10.1007/s00508-006-0693-z 
bioRxiv preprint doi: https://doi.org/10.1101/2021.02.04.429852; this version posted February 5 , 2021. The copyright holder for this preprint (which was not certified by peer review) is the author/funder, who has granted bioRxiv a license to display the preprint in perpetuity. It is made available under aCC-BY-NC-ND 4.0 International license.

54. Sapi, E.; Bastian, S.L.; Mpoy, C.M.; Scott, S.; Rattelle, A.; Pabbati, N.; Poruri, A.; Burugu, D.; Theophilus, P.A.S.; Pham, T.V.; Datar, A.; Dhaliwal, N.K.; MacDonald, A.; Rossi, M.J.; Sinha, S.K.; Luecke, D.F. Characterization of Biofilm Formation by Borrelia burgdorferi In Vitro. PLOS ONE 2012, 7, e48277. https://doi.org/10.1371/journal.pone.0048277

55. Troxell, B.; Ye, M.; Yang, Y.; Carrasco, S.E.; Lou, Y.; Yang, X.F. Manganese and Zinc Regulate Virulence Determinants in Borrelia burgdorferi. Infect. Immun. 2013, 81, 2743-52. https://doi.org/10.1128/IAI.00507-13

56. Wang, P.; Yu, Z.; Santangelo, T.J.; Olesik, J.; Wang, Y.; Heldwein, E.; Li, X. BosR Is A Novel Fur Family Member Responsive to Copper and Regulating Copper Homeostasis in Borrelia burgdorferi. J. Bacteriol. 2017, 199, e00276-17. https://doi.org/10.1128/JB.00276-17

57. Aguirre, J.D.; Clark, H.M.; McIlvin, M.; Vazquez, C.; Palmere, S.L.; Grab, D.J.; Seshu, J.; Hart, P.J.; Saito, M.; Culotta, V.C. A ManganeseRich Environment Supports Superoxide Dismutase Activity in a Lyme Disease Pathogen, Borrelia burgdorferi. J. Biol. Chem, 2013, 288, 846878. https://doi.org/10.1074/jbc.M112.433540

58. Brown, G.; Broxham, A.H.; Cherrington, S.E.; Thomas, D.C.; Dyer, A.; Stejskal, L.; Bingham, R.J. Expression, Purification and Metal Utilization of Recombinant SodA from Borrelia burgdorferi. Protein Expr. Purif. 2019, 163, 105447. https://doi.org/10.1016/j.pep.2019.105447

59. Wang, P.; Lutton, A.; Olesik, J.; Vali, H.; Li, X. A Novel Iron- and Copper-Binding Protein in the Lyme Disease Spirochaete. Mol. Microbiol. 2012, 86, 1441-51. https://doi.org/https://doi.org/10.1111/mmi.12068

60. Zhou, L.; Dhopeshwarkar, N.; Blumenthal, K.G.; Goss, F.; Topaz, M.; Slight, S.P.; Bates, D.W. Drug Allergies Documented in Electronic Health Records of a Large Healthcare System. Allergy 2016, 71, 1305-13. https://doi.org/10.1111/all.12881

61. Macy, E.; Poon K-Y, T. Self-Reported Antibiotic Allergy Incidence and Prevalence: Age and Sex Effects. Am. J. Med. 2009, 122 , 778.e1-7. https://doi.org/10.1016/j.amjmed.2009.01.034

62. Cerar, T.; Ogrinc, K.; Cimperman, J.; Lotrič-Furlan, S.; Strle, F.; Ružić-Sabljić, E. Validation of Cultivation and PCR Methods for Diagnosis of Lyme Neuroborreliosis. J. Clin. Microbiol. 2008, 46, 3375-9. https://doi.org/10.1128/JCM.00410-08

63. Busch, U.; Hizo-Teufel, C.; Boehmer, R.; Fingerle, V.; Nitschko, H.; Wilske, B.; Preac-Mursic, V. Three Species of Borrelia burgdorferi Sensu Lato (B. burgdorferi Sensu Stricto, B. afzelii, and B. garinii) Identified from Cerebrospinal Fluid Isolates by Pulsed-Field Gel Electrophoresis and PCR. J. Clin. Microbiol. 1996, 34, 1072-8. https://doi.org/10.1128/JCM.34.5.1078.1996

64. Fingerle, V.; Schulte-Spechtel, U.C.; Ruzic-Sabljic, E.; Leonhard, S.; Hofmann, H.; Weber, K.; Pfister, K.; Strle, F.; Wilske, B. Epidemiological Aspects and Molecular Characterization of Borrelia burgdorferi s.l. from Southern Germany with Special Respect to the New Species Borrelia spielmanii sp. nov. Int. J. Med. Microbiol. 2008, 298, 279-90. https://doi.org/10.1016/j.ijmm.2007.05.002 